\title{
LA MODALIDAD \\ (CON ESPECIAL REFERENCIA A LA LENGUA ESPAÑOLA)
}

\author{
Concepción Otaola Olano
}

\section{IntRoducción. Enunciación y Modalidad.}

Son muy diversas las definiciones que se han hecho de la enunciación. Para este estudio partimos de la enunciación como el acto de producción de un enunciado, el proceso complejo que lo engendra o, dicho de otra manera, el acto individual de utilización de la lengua, opuesto al enunciado, producto de esta enunciación. Por tanto, en líneas generales, el análisis de la enunciación consistirá en el estudio de las huellas del sujeto enunciador en el enunciado.

Los estudios de la enunciación han sufrido diversos vaivenes, pues si bien se remontan a la antigüedad clásica (Aristóteles, etc.), sin embargo han estado relegados de los estudios lingüísticos durante grandes períodos de tiempo.

Hoy en día ya se reconoce la llamada lingüistica de la enunciación. Para muchos lingüistas contemporáneos es evidente que las cuestiones del sujeto enunciador ya no se pueden mantener fuera del campo de la lingüística, como defendía Saussure. Así pues, presenciamos un nuevo período de interés por la enunciación, un "redescubrimiento" del lugar del sujeto en la lingüística. El auge de los estudios de la enunciación, existente en la actualidad, tuvo su inicio hacia finales de los años cincuenta en Francia con los articulos de R. Jakobson y de E. Benveniste ${ }^{1}$.

1 JAkobson, R., "Los conmutadores, las categorías verbales y el verbo ruso" en Ensayos de Lingüística General. Barcelona, Ariel, 1975. Originariamente en inglés Shifters, verbal categories and the Russian verb. Redactado en Cambridge, Mass., en 
Debemos tener presente que el término enunciación se ha tomado en distintos sentidos y lo que se entiende por tal es muy variable según las corrientes teóricas.

El concepto de enunciación más generalizado es el que la plantea como el surgimiento del sujeto en el enunciado, como la actitud del sujeto hablante con respecto a su enunciado, como la relación que el locutor mantiene por medio del texto con su iriterlocutor.

Para este estudio se parte de esta concepción general de enunciación. Concretamente se basa en la definición de $\mathrm{E}$. Benveniste ${ }^{2}$, quien considera que "La enunciación es la puesta en funcionamiento de la lengua por un acto individual de enunciación [...]. En tanto realización individual, la enunciación puede definirse, en relación con la lengua, como un proceso de apropiación. El locutor se apropia del aparato formal y enuncia su posición de locutor mediante indicios específicos". Por tanto, esta corriente intenta identificar y describir las huellas del acto en el producto. Se investigan los procedimientos lingüísticos por los que el locutor imprime su marca en el enunciado, se inscribe en el mensaje (implícita o explícitamente) y se sitúa con relación a él. Se preocupa en particular de los conectores, de las modalidades, del discurso referido, etc.

Estamos ante la enunciación desde el punto de vista de la lengua, pues, al estudiar las huellas que la enunciación deja en el enunciado, se trata un conjunto de elementos que posee la lengua y que aseguran el paso de la lengua al discurso. Nos referimos, especialmente, a la deíxis y la modalidad.

\section{LA MODALIDAD.}

Hablar de modalidad sin más precisión es exponerse a graves malentendidos. La modalidad adquiere diversas interpretaciones según provenga este concepto de la lógica, de la semántica, de la psicología, de la sintaxis, de la pragmática o de la teoría de la enunciación. Por consiguiente, el término modalidad remite a realidades muy heterogéneas como son las modalidades lógicas, las modalidades apreciativas, las modalidades de frase, las modalidades deónticas (obligación), modalidades epistémicas (probabilidad), etc.

1956 para el proyecto de Description and Analysis of Standard Russian patrocinado por el Departamento de Lenguas y Literaturas Eslavas de la Universidad de Harward. Se tradujo al francés en 1963.

Benveniste, E., "Les relations de temps dans le verbe français". Bulletin de la Société de Linguistique, LIV (1959), fasc. I.- "L'appareil formel de l'énonciation". Langages, núm. 17 (1970). págs. 12-18. Traducido al español en Problemas de Lingǘśtica General, II. Siglo XXI, ed. 1977.

2 Problemas de Lingüistica General, II, págs. 82 y sigs. 
Tres son las principales disciplinas que se ocupan de la modalidad: la lógica, la lingüística y la semiótica. Solo nos centraremos en el campo de la lingüistica. Pero, también aquí encontramos una falta de acuerdo en la definición de este concepto.

Veamos, pues, en primer lugar, los problemas de la definición de la modalidad en el terreno de la lingüística y, más particularmente, de la teoría de la enunciación.

\section{II.1. Problemas de definición. Historia.}

En un primer momento podemos tomar la modalidad como la actitud del sujeto hablante ante el oyente $y / o$ ante el contenido de la predicación emitida por él en el enunciado.

Existe una mayor o menor coincidencia entre los autores en este concepto de modalidad, pero divergen notoriamente a la hora de distinguir las principales clases de modalidad. Así lo comprobamos en un breve esbozo histórico del tratamiento de la modalidad, donde apreciamos que, para algunos autores, la modalidad se identifica exclusivamente con el modo verbal, mientras que para otros incide en toda la frase o enunciado, como la modalidad expresiva, la impresiva, etc. Para otros, se reduce a los matices del pensamiento y del sentimiento, como son la posibilidad, deseo, etc., o las diversas concepciones que el hablante tiene de lo real, irreal y posible. Por último, hay autores que combinan varios criterios.

El término modalidad se encuentra ya en Aristóteles ${ }^{8}$. Fue introducido en el vocabulario gramatical tomándolo de la lógica. Su utilización proviene, generalmente, de abordar la lengua desde una perspectiva lógico-semántica o psicológica.

Son muy escasos los gramáticos que tratan la modalidad, escasez que es patente en los estudios sobre el español, ya que, generalmente, no aparece tratada ni en las gramáticas clásicas del español ni en los estudios modernos. Una excepción son los trabajos que figuran en la bibliografía que estudian, aunque sea indirectamente, el problema de la modalidad. En este caso también se encuentra gran parte de las gramáticas del español como la de $\mathrm{J}$. Alcina y J. M. Blecua, el Esbozo gramatical de la Academia, la de Gili Gaya, etc., al exponer los tipos de oraciones (subordinadas, yuxtapuestas, etcétera). Destacamos los artículos de C. Hernández cuando aborda el problema del modo.

Los lingüistas que más se han ocupado de la modalidad son los de la

s Trata los verbos modales en De Sophisticis Elenchis. 
escuela de Ginebra como G. Galichet, A. Bonnard, F. Brunot, Ch. Bally. En todos se encuentra un sesgo psicológico o lógico-semántico. Por ejemplo, G. Galichet escribió La Grammaire Psicologique y Brunot La pensée et la langue.

Galichet identifica modo y modalidad. Considera a esta última como una categoria verbal añadida a las de aspecto, tiempo y voz. Para él la modalidad expresa la actitud del sujeto hablante con respecto a la realización. Se expresa esencialmente por el modo.

Para Bonnard "toda frase es pronunciada con vistas a informar o a ser informado, a comunicar un sentimiento o una voluntad". Así pues, la modalidad de la frase (afirmación, interrogación, exclamación, voluntad) designa una actitud adoptada por el locutor.

Para Brunot la modalidad expresa la actitud del hablante con respecto a una acción enunciada. Las ideas expresadas por la modalidad las agrupa en tres conjuntos, "como pertenecientes a una operación del juicio, del sentimiento y de la voluntad". Lingüísticamente tiene realizaciones muy diversas.

Estas ideas de Brunot fueron desarrolladas y organizadas por Bally, quien estudia la modalidad más exhaustivamente. Para Bally la modalidad es, según sus propias palabras, "la forma lingüistica de un juicio intelectual, de un juicio afectivo o de una voluntad que un sujeto pensante enuncia a propósito de una percepción o de una representación de su espíritu" ". Bally recoge de los antiguos la diferenciación entre el dictum (contenido representado) y el modus, "operación psíquica que tiene por objeto el dictum", denominada también animus loquentis o actitud del hablante.

La modalidad (o modus), pieza maestra de la frase según Bally, puede estar :

a) Explícita : cuando el verbo modal es distinto del verbo del dictum. Por ej.: "Creo que llegaré pronto".

b) Implícita : expresada por la sintaxis del dictum bajo formas diversas:

- auxiliar de modo: "Pedro debe venir".

- adverbio modal: "Quizá venga Pedro".

- modo gramatical: "Si lloviera". "Salid".

- adjetivo de juicio o de apreciación: "Esta fruta es deliciosa". "Es necesario que Pedro venga".

La gramática generativa también aborda la modalidad, aunque en sus primeros modelos, como sucede con los modelos estructuralistas anteriores,

- "Syntaxe de la modalité explicite", en Cahiers de F. de Saussure, 1942, pág. 3. 
apenas conceden un lugar al concepto de modalidad y a los conceptos lógicosemánticos que la sostienen. Con el tiempo, tras las teorias de Katz y Postal, figurará la modalidad como constituyente de frase obligatorio en la siguiente regla de rescritura.

$$
\text { Const. }\left\{\begin{array}{l}
\text { Afirm. } \\
\text { Interrog. } \\
\text { Imp. }
\end{array}\right\}+\text { (neg.) }+ \text { (enfático) }+ \text { (pasivo) }
$$

\section{II.2. Tipos de modalidad.}

Tras este somero planteamiento de la diversidad de opiniones respecto a la modalidad, podemos comprobar que ésta remite a realidades lingüísticas muy diferentes: modos gramaticales, tiempos, verbos modales, adverbios modales, tipos de oraciones (afirmación, interrogación, mandato), etc.

Para poner un poco de orden, y siguiendo en el enfoque enunciativo, podemos decir que el conjunto de hechos englobados hasta el presente bajo la denominación de modalidad podemos repartirlo ahora en dos grandes clases con relación al fenómeno de la comunicación.

- modalidades de la enunciación,

- modalidades del enunciado.

Para ello nos apoyamos en la diferencia explicada con anterioridad entre enunciación (acto que implica un proceso y dos protagonistas) y enunciado (materia enunciada, el objeto de la enunciación).

\section{II.2.1. Modalidades de la enunciación.}

El locutor al hablar adopta una actitud de cara al oyente, y los elementos lingüísticos que la manifiestan constituyen lo que denominamos modalidades de enunciación, o "modalidades de frase" de Bonnard y de Agustín García Calvo. Este último estudia estas modalidades en paralelismo con las funciones del lenguaje. Distingue cuatro funciones principales del lenguaje a las que corresponden otras tantas modalidades: expresiva, impresiva, lógica y estética. Nos interesan las tres primeras, pues son estas modalidades las que determinan la forma lingüística del enunciado (declaración, pregunta, mandato).

Una frase sólo puede recibir una modalidad de enunciación obligatoria que será declarativa, interrogativa o imperativa.

Ej.: Maria viene. „Viene Maria? Ven Maria. 
Según los estudios de André Meunier ${ }^{5}$, recogidos con posterioridad por D. Maingueneau ${ }^{6}$, la modalidad de enunciación implica una relación interpersonal. Exige, por tanto, una relación entre dos protagonistas en la comunicación y caracteriza la forma de comunicación entre locutor y oyente. Un ejemplo claro lo encontramos en los imperativos, donde se presupone la existencia del emisor y del que recibe la orden. Es sintoma de la presencia de un tú y de la interacción que se entabla alternativamente entre hablante y oyente.

II.2.2. Las modalidades de enunciado.

Si, como hemos visto, las modalidades de enunciación expresan las diversas actitudes del hablante ante el oyente, por el contrario, las modalidades de enunciado manifiestan la actitud del hablante ante el enunciado o mensaje. Explicitan la relación entre el locutor y el enunciado.

Estas modalidades de enunciado se corresponden con el concepto de modalidad propugnado por Bally, pues suponen la expresión lingüística de la actitud de un sujeto humano con respecto al contenido de una oración.

En lo que respecta a este tipo de modalidades debemos hacer unas aclaraciones :

Hay autores que limitan la modalidad a la primera persona del singular (al yo sujeto), pero otros la conciben en sentido amplio como expresión de la subjetividad de un ser humano, sujeto del enunciado, que puede ser o no el enunciador.

a) En el primer caso, es decir, cuando la modalidad se reduce a la subjetividad del yo (corriente de Bally), el sujeto modal es el sujeto de la enunciación. Puede estar expreso y coincidir con el sujeto del enunciado o puede estar implícito.

1) Para Bally, el sujeto de la enunciación o sujeto modal debe coincidir con el sujeto del enunciado. Así sucede en la modalidad explícita donde puede haber uno o dos actantes, pero un sujeto modal :

5 "Modalités et communication". Langue Française, núm. 21 (1974), págs. 8-25. "Grammaires du français et modalités. Matériaux pour l'histoire d'une nébuleuse". Drlav. Revue de linguistique, 25-1981, págs. 119-144.

- Initiation aux méthodes de Panalyse du discours. París, Hachette, 1976. Traducción esp.: Introducción a los métodos del análisis del dicurso. Buenos Aires, Hachette Argentina, 1980. 


$$
\begin{aligned}
& \text { Ej.: "Quiero llegar pronto" (un actante). } \\
& \text { "Quiero que Pedro llegue pronto" (dos actantes). }
\end{aligned}
$$

2) Posteriormente se ha demostrado que esta coincidencia no es necesaria, como se aprecia en la modalidad implícita:

Ej. : "No debía retrasarse".

"Puede que venga.

"Esta pintura es, posiblemente, la más expresiva".

b) En el caso en que la modalidad se concibe en sentido amplio como expresión de la subjetividad del sujeto del enunciado, éste puede coincidir o no con el enunciador.

$$
\begin{aligned}
& \text { Ej.: "Estoy seguro de que María vendrá" (coincide). } \\
& \text { "Pedro está seguro de que María vendrá" (no coincide. } \\
& \text { El sujeto modal es Pedro). }
\end{aligned}
$$

Por último, el hablante puede dejar su huella en un enunciado cuyo sujeto no sea el yo. Esto sucede, por ejemplo, en la diversa utilización del modo, como en las oraciones completivas:

$$
\begin{aligned}
& \text { Ej.: "Pedro no cree que hablaste". } \\
& \text { "Pedro no cree que hablaras". }
\end{aligned}
$$

En el primer ejemplo la suposición de realidad está asignada no al sujeto del verbo principal sino al hablante.

En las modalidades de enunciado se engloban tanto las modalidades lógicas como las modalidades apreciativas o subjetivas.

Las modalidades lógicas caracterizan la manera en que el sujeto presenta el predicado de la oración como verdadero, contingente (o necesario) o probable (posible), es decir, en el marco de lo real, de lo eventual o de lo irreal. Por ejemplo :

"Puede que Pedro venga". "Es posible que ya haya llegado".

Las modalidades apreciativas caracterizan la manera en que el sujeto sitúa el enunciado con relación a juicios apreciativos (de lo útil, lo triste, lo agradable, etc.), así como subjetivos (de deseo, temor, etc.). Por ejemplo:

"Está feliz de que Pablo haya venido". "Es inútil que venga".

"Temo que venga". "Siento que no venga". 
Para apreciar mejor la diferencia entre las modalidades de enunciación y las modalidades de enunciado tomemos los siguientes ejemplos:

"Estoy convencido de que Pedro ayudará a Isabel". y

"Siento que Pedro haya ayudado a Isabel".

La modalidad de enunciación es la misma en los dos ejemplos (declarativa), sin embargo, constituyen dos enunciados diferentes, contienen dos modalidades de enunciado (o modalidades en el sentido de Bally):

$$
\begin{aligned}
& \text { Estoy convencido } \ldots \ldots \ldots . . . \quad \text { (modalidad lógica) } \\
& \text { Siento } \ldots \ldots \ldots \ldots \ldots \ldots \ldots
\end{aligned}
$$

Por el contrario, dos oraciones que tienen la misma modalidad de enunciado pueden presentar dos modalidades de enunciación distintas:

"Es posible que Maria venga".

“¿Es posible que venga María?”.

La primera consiste en una modalidad de enunciación declarativa, mientras que la segunda es interrogativa. Sin embargo, ambas expresan una modalidad lógica (es posible).

\section{II.3. Medios de expresión de la modalidad.}

Uno de los factores que acrecientan la dificultad de estudio de la moda lidad es que sus medios de expresión son de una desconcertante variedad y no se dejan describir con la ayuda de las clasificaciones gramaticales o léxicas normales. Así la modalidad "posible" aparece según las opciones en estructuras sintácticas distintas:

"Es posible que Pedro venga".

"Puede que Pedro venga".

"Quizá venga Pedro".

"La venida de Pedro es posible", etc.

Vemos que la categoria gramatical de la modalidad varía sensiblemente: adjetivo, adverbio, verbo, etc.

Efectivamente, la modalidad tiene unos medios de expresión diversos. En líneas generales podemos decir que la actitud del sujeto hablante con 
respecto al enunciado y al oyente puede expresarse a través de distintos medios lingüísticos: léxicos (lexemas), gramaticales (gramemas) y fonéticofonológico (entonación). Así se aprecia en el siguiente esquema:

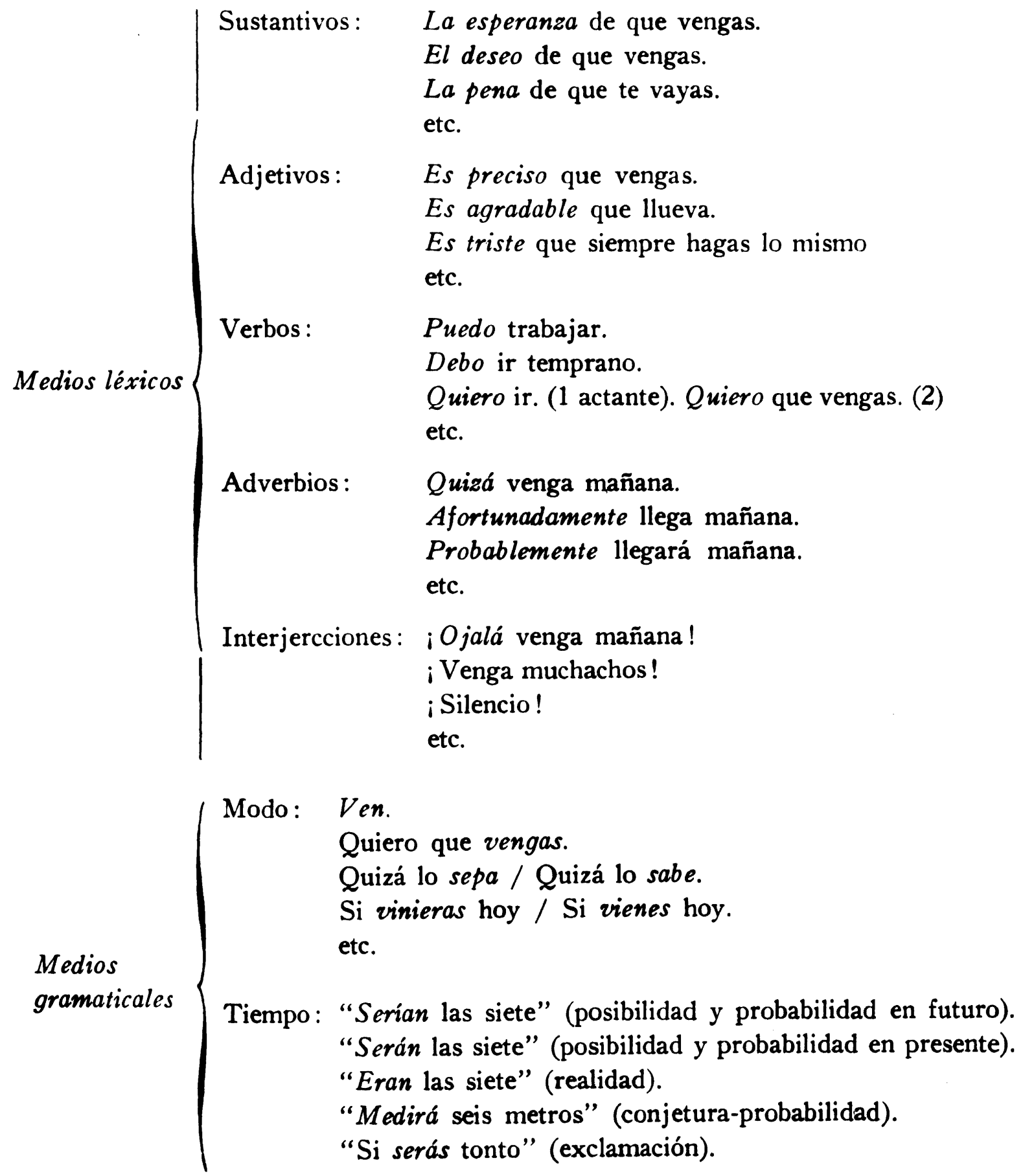

Medios fonéticos-fonológicos: Entonación. Vehículo importante de la expresión afectiva. 
Un ejemplo de la gran variedad de medios lingüísticos para expresar la modalidad, lo tenemos en la modalidad de enunciación imperativa, pues el mandato puede expresarse con formas verbales, con formas nominales (frases nominales), con fórmulas indirectas, etc.:

Medios léxicos (que pueden ser frases nominales):

- sustantivos: ¡Silencio! ;Ojo!

- adjetivos: ;Alli!

- interjecciones : ¡Hala!

Medios gramaticales

(con formas verbales que pueden ser tanto flexivas como no):

Flexivas: Cállate.

Tú te callas.
No flexivas: No fumar.

¡Andando!

¿Quietos!

Medios fonéticos-fonológicos: Entonación. Los movimientos de la entona ción traducen siempre las emociones del que habla. En el momento psíquico-afectivo del hablante de emitir una oración imperativa se originan una serie de factores fonéticos. Son relevantes las modificaciones en el timbre, tono y situación.

No tratamos ahora las fórmulas indirectas que también expresan mandato como ;Si callaras y comieras!, o interrogativas como ${ }_{6} \mathrm{Me}$ quieres dar ese libro de una vez? o \&No me ayudas un poco?

Entre las realizaciones léxicas más frecuentes de la modalidad en la lengua española destacan, por ser más problemáticas, los adverbios modales y los verbos modales. También el modo, medio gramatical, ha suscitado polémicas, pues se ha confundido o identificado con la modalidad.

Me limitaré a presentar algunas precisiones sobre estos elementos modales de la lengua española.

\section{II.3.1. Los adverbios modales.}

Como ya sabemos, los adverbios constituyen una categoría muy heterogénea. Dentro de esa heterogeneidad y acorde con el concepto de modalidad, sólo nos interesan los que realizan la función de modificadores de oración o mediatizadores de nexus. Estos son los modales de frase o indicadores de modalidad que mediatizan toda la oración, es decir, el conjunto de toda la predicación. Estos adverbios han recibido distintas denominaciones. Nilson- 
Ehle los llama "adverbios de oración"; Ana María Barrenechea, "modificadores de núcleo oracional"; Rafael Seco, "adverbios oracionales"; C. Hernández, "mediatizadores de nexus"; J. Alcina y J. M. Blecua, "modificadores de toda la frase"; los generativistas, "indicadores de modalidad" o "adverbios de frase".

Nos referimos a los adverbios afirmativos, negativos y dubitativos. Estos se diferencian de los modificadores de predicado, pues estos últimos inciden solo en el verbo o núcleo verbal. Algunos autores como Thomason ciñen el carácter de adverbios únicamente a los modificadores de predicado, siendo los otros simplemente modificadores de frase.

Las formas lingüísticas con carácter adverbial que manifiestan la modalidad son de dos tipos: lexemas (quizá, seguramente, etc.) y locuciones o frases adverbiales (por supuesto, etc.). En el primer grupo están incluidos algunos adverbios terminados en -mente, como luego veremos.

Así, en el conjunto heterogéneo de la categoria adverbial hay un grupo de elementos que modifican la frase e indican la modalidad. Se han realizado diversos estudios, como los de André Borillo, Eddy Roulet, Robert Martin ${ }^{7}$, para caracterizar este conjunto de adverbios modales, desde el punto de vista sintáctico, distribucional, estudiando el lugar que ocupan en el enunciado, pausas, combinaciones con otros adverbios, etc. Pero, en definitiva, pienso, como André Meunier, que solo criterios semánticos permiten hacer de ellos una subclase especial dentro del conjunto de adverbios.

El principal criterio que agrupa a estas formas lingüísticas es que expresan la modalidad, es decir, que son la huella de la toma en consideración de la predicación por parte del enunciador. Se les denomina "modales", "subjetivos" o de "opinión", pues expresan la opinión del enunciador con respecto a lo que enuncia.

Para la clasificación de estos adverbios modales seguiremos la distinción realizada en este trabajo entre modalidades lógicas y modalidades apreciativas. En el primer grupo se integran los adverbios asertivos afirmativos, negativos y de duda. Podemos agruparlos de acuerdo con la distinción lógica modal entre lo posible, lo real y lo necesario:

7 Borillo, A., "Les adverbes et la modalization de l'assertion". Langue Franfaise, núm. 30 (1976), págs. 74-84.

RoulET, E., "Des modalités implicites intégrées en français contemporain". Cahiers de Ferdinand de Saussure, núm. 33 (1979), págs. 41-76.

Martin, R., "La notion d'“adverbe de phrase", en Rohrer, C. y Ruwet, N. (eds.), Actes du Colloque Franco-Allemand de Grammaire Transfortionelle, II. Etudes de sémantique et autres. Tübingen, Niemeyer, 1974. 
a) Adverbios que expresan modalidades lógicas (adverbios asertivos o modificadores de la aserción).

$$
\text { “posible” } \quad\left\{\begin{array}{l}
\text { quizá } \\
\text { tal vez } \\
\text { acaso } \\
\text { probablemente } \\
\text { seguramente } \\
\text { etc. }
\end{array}\right.
$$$$
\text { "real" } \quad \begin{aligned}
& \text { sí } \\
& \text { ciertamente } \\
& \text { verdaderamente } \\
& \text { naturalmente } \\
& \text { evidentemente } \\
& \text { efectivamente } \\
& \text { inevitablemente } \\
& \text { por supuesto } \\
& \text { desde luego } \\
& \text { sin duda alguna } \\
& \text { indiscutiblemente } \\
& \text { indudablemente } \\
& \text { etc. }
\end{aligned}
$$

“necesario" $\left\{\begin{array}{l}\text { necesariamente } \\ \text { obligatoriamente } \\ \text { forzosamente } \\ \text { etc. }\end{array}\right.$

\begin{tabular}{l|l} 
asertivos & no \\
de ninguna manera \\
en modo alguno \\
en absoluto \\
etc.
\end{tabular}

b) Adverbios que expresan modalidades apreciativas (adverbios evaluativos o apreciativos).

$$
\left\{\begin{array}{l}
\text { afortunadamente } \\
\text { desgraciadamente } \\
\text { curiosamente } \\
\text { por suerte } \\
\text { desafortunadamente } \\
\text { paradógicamente } \\
\text { etc. }
\end{array}\right.
$$

Los adverbios evaluativos se diferencian de los asertivos en que los primeros son factivos, es decir, presuponen la verdad de la predicación. 
II.3.1.1. Algunas puntualizaciones sobre estos adverbios modales.

Los adverbios en -mente que realizan esta función de mediatizador de nexus, por lo general, se pueden transformar en oraciones atributivas.

$$
\begin{aligned}
& \text { Ej.: "Evidentemente el tren no llegó a su hora". } \\
& \text { "Es evidente que el tren no llegó a su hora". }
\end{aligned}
$$

Los adverbios asertivos pueden funcionar como pro-oracionales con un valor equivalente a una oración, especialmente en la respuesta a una pregunta total. Son frases nominales.

$$
\begin{aligned}
\text { Ej.: “¿Está dominada la situación? } & \\
& \text { Efectivamente o ciertamente o si (está dominada) o no }
\end{aligned}
$$

Precisamente esta facultad propia de ciertos adverbios lleva a algunos autores a negar su carácter de adverbios. Por el contrario, otros, como A. Borrillo $^{8}$, consideran que es una característica propia de estos adverbios de frase, aunque puntualiza que no sucede en la respuesta a cualquier pregunta total, sino que hay algunas restricciones.

No debemos olvidar los 12 criterios que presenta Ole M $\phi$ rdrof ${ }^{\theta}$ para caracterizar los adverbios en -mente franceses como adverbios de frase. Algunas de estas características también las ha estudiado D. de RodríguezPasqués ${ }^{10}$ con respecto a los adverbios en -mente españoles en función de modificadores de oración. Presenta los siguientes caracteres:

Caracteres formales:

1) Modifican a toda la oración. Pueden transformarse en oraciones impersonales.

2) Se ubican al principio de la oración, o se intercalan entre comas en la lengua escrita. En el habla se señalan por la entonación.

3) Son raros en oraciones de modo subjuntivo o imperativo.

4) Es construcción corriente en el habla familiar, aunque su número en este caso es limitado.

5) No admiten otro adverbio que los modifique.

8 Op. cit., pág. 75.

- "Sur la classification des adverbes en -ment". Revue Romane, XI-2, 1976, páginas 317-333.

10 "Morología y Sintaxis del adverbio en -mente". Actas del Tercer Congreso Internacional de Hispanistas. Colegio de México, 1970, págs. 293-303.

LXVIII, $1 .^{\circ}-2 .^{\circ}-8$ 
Caracteres semánticos:

1) Generalmente son intensivos (de duda, de afirmación, de negación, etcétera), pero también pueden ser de restricción o limitativos.

2) La expresión parentética del tipo "francamente hablando" o "técnicamente hablando" se relaciona estrechamente al adverbio modificador de oración. Por economía verbal se suprime el gerundio.

3) Cuando obedecen a intención estilística invierten el orden de las palabras. Aparecen en primer término para realzar un elemento oracional.

Por otra parte, autores como Estanislao Ramón Trives ${ }^{11}$, y especialmente generativistas como Lakoff, demuestran cómo estos adverbios son modalidades de la enunciación o fuerza performativa, pues afirman que, en oraciones como, por ejemplo, "Seguramente tu amigo no te habia dicho la verdad", nos inducen a pensar en una modalidad de la enunciación del tipo " "Declaro con relativa seguridad / probabilidad" que tu amigo no te habia dicho la verdad".

En este aspecto debemos recordar la diferenciación establecida por algunos generativistas en los "adverbios de frase" entre los perfomativos (francamente, sinceramente, etc.) que pertenecen al plano de la enunciación y los de actitud (probablemente, evidentemente, etc.) que pertenecen al enunciado.

Otra aclaración que debemos hacer es que los adverbios si y no pueden incidir en el enunciado o en la enunciación. Por ejemplo, en la pregunta “¿No quieres jugar?”, la respuesta si significa si quiero. En este caso no se atiende al enunciado (lo que se dice) sino a la enunciación (el decirlo). Se afirma la expresión del contenido del enunciado y no el contenido.

Otro ejemplo lo tenemos en la combinación de no con los verbos de opinión como creer. En "creo haber traído el libro" puede haber dos negaciones :

1) creo no haber traído el libro,

2) no creo haber traido el libro.

La diferencia estriba en la negación del enunciado (en el primer caso se niega el contenido del hecho, haberlo traído) y en la negación de la enunciación (en el segundo caso se niega la expresión de ese contenido).

11 Estudios sintácticos-semánticos del español. I. Dinámica interoracional. Murcia, Edit. Godoy, 1982. 


\section{II.3.2. Verbos modales.}

El verbo en cuanto expresión de la modalidad ha suscitado muchas polémicas. Estas se derivan de cuestionarse, en primer lugar, si existen los verbos modales. Si la respuesta es positiva, nuevas interrogantes se plantean : ¿Cuántos y cuáles son?, ¿Qué función desempeñan?, es decir, ¿son o no auxiliares?

El resultado es la existencia de posturas muy contradictorias que van desde la afirmación de que todo verbo subjetivo es susceptible de ser empleado modalmente, como dice B. Pottier ${ }^{12}$, hasta concebir como modales únicamente dos verbos : poder y deber, o tres : deber, poder y soler. Esta última postura es defendida, entre otros, por Juan Alcina y José Manuel Blecua ${ }^{13}$, así como por Antonio Narbona ${ }^{14}$, quien añade osar $y$, con reservas, acostumbrar.

$\mathrm{Si}$ bien en lo que se refiere a la lengua española hay una generalidad de gramáticos (Gili Gaya, La RAE, Amado Alonso y P. Henríquez Ureña) que reconocen como verbos modales, principalmente, los verbos deber, poder, saber, querer (y soler); sin embargo, también admiten que la lista se puede ampliar con sus sinónimos y con los verbos que expresan deseo, voluntad, comportamiento, etc. Algunos, como D. Bolinger ${ }^{15}$, también incluyen las expresiones perifrásticas verbales haber de y tener que.

Los verbos modales suelen estudiarse en estrecha relación con los denominados auxiliares. Se les ha considerado como "semiauxiliares de modo" (Roca Pons y Casado Velarde ${ }^{16}$ ), "auxiliares modales" (Hadlich), "auxiliares fuertes" (S. Skydsgaard), etc. Otros autores niegan a los verbos modales su carácter de verbos especiales. Klein ${ }^{17}$ los considera como verbos principales en la estructura profunda. Autores como R. P. Stockwell, Bowen y $\mathrm{R}$. Martin niegan la existencia de auxiliares modales, aunque reconocen la peculiaridad de poder, deber y querer.

No se van a tratar aquí las distintas teorías sobre los modales (auxiliares o no), pero, ateniéndonos al sentido amplio de verbo modal, es decir, el

12 "Les voix du français. Sémantique et syntaxe". Cahiers de Lexicologie, 1978-2.

13 Gramática española. Barcelona, Ariel, 1983.

14 “¿Verbos modales en español?". Verba, 8 (1981), págs. 171-186.

15 "Modes of Modality in Spanish and English". Romance Philology, v. XXIII, 4, 1970, págs. 572-586.

16 Roca Pons, J., Introducción a la gramática. Barcelona, Teide, 1985.

Casado Velarde, M., Estudio de los verbos de modalidad en La Familia de Pascual Duarte. Resumen de Tesis de Licenciatura defendida en 1972 en la Universidad de Sevilla.

17 Modal auxiliaries in Spanish. Reprinted University Microfils International. Ann Arbor, London, 1985. Original: "Modal auxiliaries in Spanish". Studies in Linguistics and Language Learning. Vol. IV, Seatle, 1968. 
que expresa la posición del hablante ante los hechos que enuncia, podemos caracterizarlos, a grandes rasgos, funcional y semánticamente.

Funcionalmente prevalece la teoría de que son un tipo de "auxiliares" o "semiauxiliares". La expresión de la modalidad verbal con estos verbos exige la presencia de dos verbos: el verbo de modalidad y el verbo del dictum. No solo forman una única oración con un actante como poder y deber: "Puedo cantar", sino que pueden ser dos cláusulas subyacentes con dos actantes: "Quiero que vengas".

Semánticamente, puesto que la modalidad es una categoria esencialmente semántica que se muestra plenamente en unos pocos verbos (poder, deber) $y$, en distinto grado, en otros muchos, podremos clasificar los verbos modales de acuerdo con los tipos de modalidades que hemos distinguido:

\section{a) Verbos modales en sentido limitado.}

Verbos inequivocamente modales (según ciertos autores: poder, deber, soler).

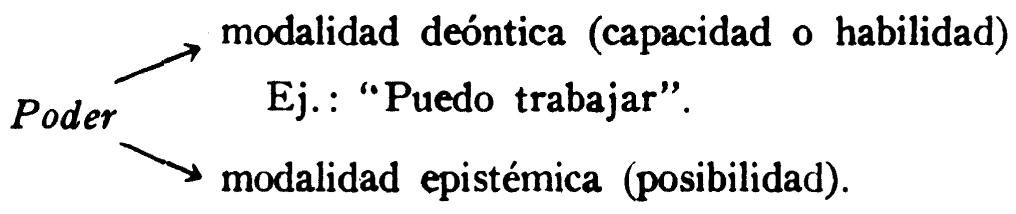

Ej.: "Puede que llueva".

"Con este frío puedo coger un resfriado".

modalidad deóntica (obligación).
Ej.: "Debo regresar cuanto antes".
Ejodalidad epistémica (posibilidad). "Debí llegar tarde porque ya había salido el tren".

Soler $\longrightarrow$ Son muchos los autores que le niegan el carácter de verbo modal. Lo consideran más aspectual.

Hay autores que deslindan un tercer sentido en los verbos modales poder y deber. Poder = dar permiso ("Pedro puede venir"); Deber = dar una orden ("Pedro debe venir"). Afirman que precisamente en este tercer sentido se incluye de manera más directa al locutor. Al igual que el valor episté- 
mico, este tercer valor es subjetivo, puesto que el locutor es el que "obliga, permite o prohibe".

Por el contrario, otros autores consideran que en los verbos poder y deber únicamente el empleo epistémico es el auténtico empleo modal.

Por último, algunos autores niegan la necesidad de este desdoblamiento en los verbos modales ${ }^{18}$.

b) Verbos modales en sentido amplio.

b.1. Verbos que expresan modalidades lógicas:

Verbos de opinión ligados a lo intelectual, a operaciones de inteligencia : creer, suponer, pensar, sospechar, dudar, temerse, etc.

b.2. Verbos que expresan modalidades apreciativas:

- Verbos de voluntad: querer, desear, intentar, mandar, ordenar, rogar, esperar, pretender, pensar (tener intención de), etc.

- Verbos de sentimiento: sentir, doler, alegrarse, lamentar, molestar, etc.

\section{II.3.3. Modo y modalidad.}

Siempre ha habido una tendencia a confundir o identificar modo y modalidad, pero se puede demostrar que el modo es una forma más de expresar la modalidad y que la modalidad no es privativa de los modos verbales, aunque tradicionalmente asi se ha considerado. La identificación del modo con la modalidad ha sido propiciada por la consideración del modo, ya desde los gramáticos clásicos, como la "actitud mental del hablante". Pero, como ya dijimos anteriormente, existen las modalidades de enunciación y las moda-

18 Para toda esta problemática puede consultarse:

Bolinger, D., "Modes of Modality in Spanish and English". Op. cit.

Fieldman, D. M., "Some Structural Characteristics of the Spanish modal verb phrase". Boletin de Filología. Tomo XVI-1964, págs. 241-255.

Rivero, M. a L., "El modo y la presuposición” en Estudios de Gramática Generativa del español. Madrid, Cátedra, 1979.

- "La ambigüedad de los verbos modales: una visión histórica”. Revista Española de Lingüística. Año 5, fasc. 2, 1975, págs. 401-442.

Roulet, E., "Des modalités implicites integrées en français contemporain". Op cit. Solano-Araya, J. M., Modality in Spanish: An Account of Mood. Ann Arbor, Michigan, Univ. Mic. I. 1984. 
lidades de enunciado, diferencia basada en que esta actitud esté orientada hacia el oyente o hacia el enunciado.

Comprobamos que no todos los modos entran dentro del mismo tipo de modalidad, pues, por ejemplo, el imperativo, para algunos autores que lo reconocen como modo independiente del subjuntivo, corresponde únicamente a las modalidades de enunciación, mientras que el indicativo y el subjuntivo a las modalidades de enunciado.

Para comprender mejor la diferencia entre modo y modalidad partiremos del concepto de modo. Sabemos que este concepto no tiene una validez única y general, sino que abunda la diversidad de opiniones y criterios. Estos se abren en un abanico que va desde la consideración de la inflexión del verbo como base distintiva $\mathrm{y}$, por consiguiente, considerando al subjuntivo como modo de la subordinación, hasta el criterio de la actitud mental del hablante ante el enunciado y el oyente. Son defensores de este último criterio autores como Gili Gaya, Alarcos Llorach, Pottier, C. Hernández, etc. Pero siguiendo nuestro enfoque enunciativo podemos perfilar más el concepto de modo para diferenciarlo del de modalidad.

Para ello nos basamos en los trabajos de autores como Agustín García Calvo, Sebastián Mariner, Lisardo Rubio, César Hernández, etc., quienes intentan superar el criterio único de noción básica del modo.

Tanto A. García Calvo ${ }^{10}$ como S. Mariner ${ }^{20}$ propugnan una triple noción básica en la categoria modal y formulan los tres criterios siguientes como distintivos del modo:

1. Modo como forma lingüística capaz de manifestar la modalidad.

2. Modo como expresión de la actitud del hablante frente al enunciado.

3. Modo como indicador de subordinación.

Nos detenemos en los dos primeros criterios por dos razones: porque inciden directamente en la modalidad y porque el último, como han demostrado S. Mariner y C. Hernández ${ }^{21}$, no es criterio distintivo y básico de la categoría modal en el verbo español.

\footnotetext{
10 "Preparación a un estudio orgánico de los modos verbales sobre el ejemplo del griego antiguo". Emérita, 1957, XXV, pags. 449-486.

"Funciones del lenguaje y modalidades de la frase". Estudios Clásicos, 4, 329-350.

20 "Triple noción básica en la categoría verbal castellana". Revista de Filología Española. T. LIV-1971, págs. 209-252.

21 C. Hernindez, Gramática funcional del español. Madrid, Gredos, 1984.
} 


\section{Modo como forma lingüistica capaz de manifestar la modalidad.}

Se corresponde con las anteriormente explicadas modalidades de frase o de enunciación. El modo en este caso traduce el tipo de comunicación instituida por el locutor entre él y su interlocutor (relación locutor-oyente).

Se relaciona con tres funciones principales del lenguaje: expresiva, impresiva y lógica. Cada una de estas funciones comporta unas modalidades. Así, la impresiva supone las modalidades yusiva-imperativa, votiva $\mathbf{u}$ optativa; la lógica abarca las oraciones enunciativas, interrogativas, etc. La $e x-$ presiva, presente en las exclamativas, es una exageración expresiva de las tres modalidades básicas: afirmación, interrogación $\mathrm{y}$ orden.

En lo que se refiere a los modos del verbo español, las modalidades dominantes son correlativas a las tres funciones del lenguaje de Bühler, de representación, expresión, apelación o, como hemos dicho antes, lógica, expresiva e impresiva.

No se pretende tratar a fondo los modos. Unicamente, como ejemplo, vamos a ver las formas verbales que expresan estas modalidades.

El llamado imperativo es la única forma verbal marcada exclusivamente por la modalidad impresiva que responde a la función conativa o apelativa.

Aunque las demás formas verbales no poseen una modalidad exclusiva, sin embargo, apreciamos que unas formas son más propias que otras para recibir determinadas modaliclades. Así la aseveración afirmativa o negativa y la interrogación estarán expresadas en Indicativo:

$$
\text { Ej.: "Pablo viene”. “¿Viene Pablo?”. }
$$

Por tanto, el indicativo es un modo objetivo en el que predomina la función representativa. Es el modo de la factividad ${ }^{22}$ y la aseveración directa. Por consiguiente, su modalidad distintiva es la declarativa (o lógica), aunque, dado que ninguna forma verbal expresa exclusivamente una modalidad, también aparece el Indicativo en las modalidades de frase negativa e interrogativa.

Un ejemplo de la no exclusividad de modalidades para las formas verbales, es el hecho de que el Indicativo puede adquirir una modalidad impresiva, sintagmáticamente, como valor en el decurso:

$$
\text { Ej.: "No matarás" y "Tú te callas". }
$$

Es importante recalcar que estas modalidades de la enunciación o modalidades de frase son las que reflejan la actitud del hablante ante el oyente.

22 Concepto propuesto por P. KIPARSKY y C. KIPARSkY en 1971 y que indica que se presupone la verdad de la predicación. 
Algunos autores, al no hacer la diferenciación entre modalidades de la enunciación (actitud del hablante ante el oyente) y modalidades del enunciado (actitud del hablante ante el enunciado), constriñen "la actitud del hablante" a una de estas modalidades.

Asi, por ejemplo, Lisardo Rubio ${ }^{23}$ distingue, en el modo verbal, el dictum y el animus loquentis. El dictum corresponde a las formas verbales de indicativo, subjuntivo, las cuales expresan las nociones de "real", "posible" e "irreal"; mientras que el modus o actitud mental del hablante viene expresado en las modalidades de la frase: afirmativa, interrogativa e impresiva, que desde fuera inciden sobre el verbo. Explica el modo con la fórmula modo $\times$ modalidad. Así pues, para L. Rubio la actitud del hablante está manifestada únicamente en las modalidades de la frase. No concibe la actitud del hablante en el nivel de la forma verbal.

Por el contrario, para $C$. Hernández la actitud del hablante se encuentra en el nivel de la forma, es decir, en el segundo criterio anteriormente propuesto: el modo como expresión de la actitud del hablante frente al enunciado. Así pues, aunque considera lo que él denomina modalidad, es decir, las modalidades de frase, como factor distintivo de los modos, sin embargo, este criterio, para él, es secundario e implicado y superpuesto al de "actitud del hablante" (o lo que venimos denominando modalidades de enunciado).

2. El modo como expresión de la actitud del hablante frente al enunciado.

Manifiesta la relación hablante-enunciado. Por tanto, el modo expresa la subjetividad o la "representación mental" y aun la "irrealidad". Abarca las modalidades lógicas y las apreciativas.

El subjuntivo es el modo que proyecta la presencia del hablante en el enunciado, ya que expresa la subjetividad del hablante o del sujeto de la comunicación ante el enunciado.

Ej.: "Temo que venga mañana".

Es el modo de la no factividad y de la posibilidad.

La duda, la posibilidad y la probabilidad se expresan en subjuntivo o en indicativo según la situación con respecto a la afirmación o negación absoluta y a la duda absoluta. Así el modo subjuntivo expresa la duda absoluta, mientras que la posibilidad y probabilidad pueden ir en indicativo, pues depende del grado.

28 "Los modos verbales latinos". Emérita, 36, 1968, págs. 77-96. 


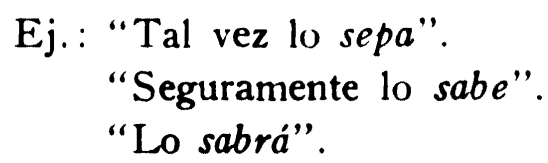

Que el modo expresa la actitud del hablante frente al enunciado lo apreciamos claramente en los siguientes ejemplos:

"Está decidido a marcharse aunque hoy vienen sus amigos".

"Está decidido a marcharse aunque hoy vengan sus amigos".

"Está decidido a marcharse aunque hoy vinieran sus amigos".

El hablante se comporta ante el hecho de la venida de sus amigos como de la decisión en el primer caso, no conocimiento de si vienen o no en el segundo y conocimiento de que no vienen, en el tercero.

Como conclusión al tema del modo y la modalidad, podemos afirmar con C. Hernández ${ }^{24}$ que "son dos los criterios básicos de la categoria del modo castellano": 1) la modalidad (de la frase) y 2) la actitud del hablante. Pero, tras esta exposición, quizá sea conveniente precisar que la actitud del hablante está presente en ambos: orientada hacia el oyente, con lo que mostraría las que he llamado modalidades de frase o modalidades de enunciación, es decir, el punto 1 , o bien esa actitud del hablante puede estar orientada hacia el enunciado (las modalidades del enunciado) y, entonces, se correspondería con el punto 2 , es decir, la actitud del hablante según $\mathrm{C}$. Hernández.

Este estudio queda limitado en un doble frente: por una parte, el hecho de que se traten únicamente algunas realizaciones de la modalidad; por otra, el ceñirse a esbozar la problemática de cada una de ellas. Es importante destacar que, en este trabajo, solo se ha pretendido observar la modalidad teniendo en cuenta las teorías enunciativas. Se comprueba que éstas pueden ser de gran utilidad a la hora de abordar ciertos aspectos gramaticales de la lengua.

24 Op. cit., pág. 290. 